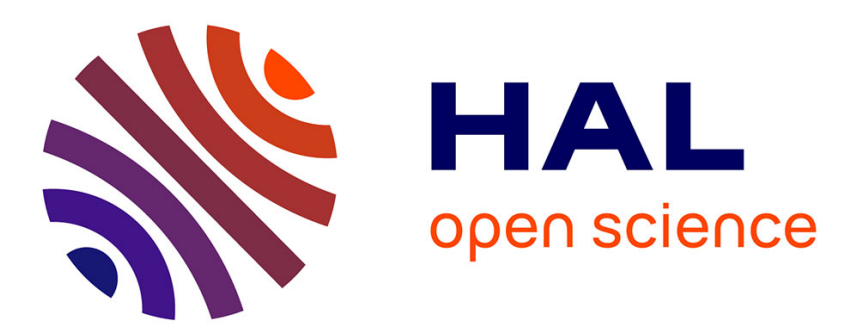

\title{
Les syndicats mixtes de transport de la loi SRU: un outil pour la gouvernance interterritoriale des mobilités?
}

Cyprien Richer, Sophie Hasiak, Nicolas Jouve

\section{To cite this version:}

Cyprien Richer, Sophie Hasiak, Nicolas Jouve. Les syndicats mixtes de transport de la loi SRU: un outil pour la gouvernance interterritoriale des mobilités?. Flux - Cahiers scientifiques internationaux Réseaux et territoires, 2011, 83, pp.51-67. halshs-00608749

\section{HAL Id: halshs-00608749 \\ https://shs.hal.science/halshs-00608749}

Submitted on 14 Jul 2011

HAL is a multi-disciplinary open access archive for the deposit and dissemination of scientific research documents, whether they are published or not. The documents may come from teaching and research institutions in France or abroad, or from public or private research centers.
L'archive ouverte pluridisciplinaire HAL, est destinée au dépôt et à la diffusion de documents scientifiques de niveau recherche, publiés ou non, émanant des établissements d'enseignement et de recherche français ou étrangers, des laboratoires publics ou privés. 


\title{
LES SYNDICATS MIXTES DE TRANSPORT DE LA LOI SRU : UN OUTIL POUR LA GOUVERNANCE INTERTERRITORIALE DES MOBILITES ?
}

\author{
Cyprien RICHer (1), Sophie HASIAK (2), Nicolas Jouve (3)
}

CETE Nord-Picardie, Ministère de l'Ecologie, du Développement Durable, des Transports et du Logement. Pôle de Compétences et d'Innovations CERTU « Transports du quotidien ».

(1) Chargé de Recherches

(2) Directrice d'Etudes

(3) Chargé d'Etudes

CETE Nord-Picardie

2 rue de Bruxelles, BP 275

59019 Lille Cedex

Correspondant :

cyprien.richer@developpement-durable.gouv.fr

\section{Résumé}

En 2000, la loi "Solidarité et Renouvellement Urbain" (SRU) a encouragé le développement de syndicats mixtes de transport dit "SRU " sous une forme institutionnelle nouvelle pour favoriser la coopération entre Autorités Organisatrices de Transport (AOT) de différents niveaux. Une dizaine d'années après, cet article propose d'étudier ces structures syndicales pour tirer un bilan des situations existantes et ouvrir des débats sur l'application locale de la réforme. Devant le défi croissant de faire interagir les politiques urbaines au-delà des clivages sectoriels ou spatiaux, les syndicats mixtes de transport SRU constituent-ils un véritable enjeu pour une nouvelle gouvernance des mobilités, susceptible de mieux prendre en compte "l'interterritorialité » des enjeux contemporains ? Le premier bilan de l'étude des syndicats mixtes SRU témoigne de la souplesse de l'outil face à la diversité des situations locales. Ces syndicats apparaissent comme un fragile rouage de l'architecture interterritoriale malgré l'étendue des enjeux qu'ils portent en matière d'intermodalité et de "mobilité durable ". II convient cependant de relativiser les interprétations par rapports aux récentes évolutions et créations de syndicats SRU non intégrées dans cette étude. 


\section{INTRODUCTION. Les syndicats mixtes de transport, objets de recherche sur la gouvernance des mobilités}

Face aux défis considérables engendrés par l'explosion des mobilités et aux besoins croissant « de politiques multi-niveaux » (Jemelin, Vodoz, Pfister, 2004), la loi « Solidarité et Renouvellement Urbains ${ }^{1}$, adoptée en 2000 , s'inscrit dans une démarche de renforcement de la cohérence spatiale et intersectorielle des collectivités locales. Afin de faciliter la coordination multiéchelles des autorités responsables des transports, la loi a notamment encouragé le recours à des syndicats mixtes d'une nouvelle génération regroupant différents niveaux d'autorités organisatrices de transport.

Cet article porte sur les syndicats mixtes de transport de la loi SRU qui, en cherchant à favoriser les coordinations institutionnelles, constitue un « objet d'étude stimulant » (Kaufmann, Pattaroni, 2010). Ces réflexions qui prolongent deux travaux de recherche, l'un pour le PREDIT (Gallez, Menerault, 2005) et l'autre pour le CERTU (Richer, Menerault et al., 2007), alimentent les débats sur les modalités d'organisation des institutions locales, ici dans le domaine des transports. En particulier, l'objet de recherche que constitue la nouvelle formule de syndicat mixte de transport, questionne directement la "gouvernance des mobilités ". Cet enjeu est de taille car toutes les métropoles en tant qu'écosystèmes "polycentriques » et "pluralistes " (Pinson, 2010) sont soumises à une capacité d'arbitrage restreinte voire absente ${ }^{2}$. Ainsi, on peut penser que la maîtrise de questions complexes telle que la mobilité passe par la gouvernance.

Plusieurs questions guident cette analyse des syndicats mixtes de transport : Dans quelle mesure "l'innovation institutionnelle » dont est porteuse la loi SRU, constituent-elle un instrument en faveur d'une meilleure prise en compte de "l'interterritorialité " des enjeux contemporains ? Ces syndicats mixtes «SRU » participent-ils à la transformation des modalités d'organisation des transports publics, marquées traditionnellement par un partage strict des rôles et des périmètres ? Est-ce que l'adage qui unifie fermement depuis les années 1970, un réseau (de transport collectif), un périmètre (le PTU, Périmètre de Transport Urbain), une institution (l'AOTU, Autorité Organisatrice de Transport Urbain) et une source de financement sectorielle (le VT, Versement-Transport) évolue au profit de nouvelles modalités de coopération?

Cet article se décompose en différentes approches :

- bibliographique et chronologique d'abord (Partie 1), avec un état de l'art des formes d'organisation des politiques publiques qui opposent gouvernement (capacité d'arbitrage) et gouvernance (coopération). Cette tension s'observe au fil des réformes territoriales dans l'évolution des formules d'AOTU oscillant entre « gouvernement » et « gouvernance ";

- comparative ensuite (Partie 2), à travers l'analyse des 7 syndicats mixtes SRU opérationnels en 2008. L'article exploite une étude du CETE Nord-Picardie (Hasiak, Jouve, 2010a) qui permet de tirer un premier bilan de ce nouvel outil de coopération et de gouvernance locale. Huit ans après sa mise en place réglementaire, ce panorama permet notamment d'observer les facteurs de succès et les principales difficultés rencontrées.

- analytique enfin (Partie 3), avec l'identification de questions clés permettant d'ouvrir des perspectives sur ces nouvelles formes de coopération. Cette étude soulève au moins trois débats sur la gouvernance des mobilités: le premier sur sa complexité nouvelle et le nouveau positionnement de ses acteurs, le deuxième sur la question spatiale et le troisième sur le rôle présent et futur des syndicats mixtes SRU dans le « management des mobilités durables ». 


\section{PARTIE 1. "Gouvernement " contre " gouvernance » : les transports collectifs face aux l'organisation des politiques publiques}

\section{1/ La "gouvernance urbaine " et les approches "interterritoriales »: nouveaux paradigmes des politiques publiques?}

Cette étude bibliographique non exhaustive témoigne de l'ambivalence des modalités d'organisation des politiques d'agglomération. En simplifiant les débats sur les politiques publiques en milieu urbain, il apparaît que deux formes d'organisation se font face : d'un côté, le « gouvernement » et de l'autre la « gouvernance » (Wiel, 2010).

La formule du gouvernement urbain correspond à un régime intégré en général central et métropolitain ayant une forte capacité de décision et d'arbitrage. Assis sur une aire suffisamment large, le gouvernement, tel "Gargantua », " attrape (ou devrait attraper) tous les problèmes, toutes les compétences dans son territoire d'élection » (Estèbe, 2008).

Ces dernières années, malgré «le renforcement de leurs capacités techniques et politiques à concevoir et mettre en œuvre des politiques publiques" (Pinson, 2010), les gouvernements locaux disposent encore d'une capacité d'arbitrage réduite. En effet, les lois de décentralisation " ont laissé inchangé un principe de compétence générale » qui permet " à chaque niveau d'intervenir dans une très grande variété de secteurs » (Pinson, 2010). La "prolifération organisationnelle ${ }^{3}$ ou la "multiplication des acteurs intervenant dans la fabrique des politiques urbaines " se traduit par une dispersion des ressources pour l'action (Pinson, 2006). En outre, l'émergence d'un gouvernement des grandes villes est confrontée à « l'institutionnalisation d'un régime périurbain, assumant sa spécificité et vivant très largement de la mobilité (professionnelle et résidentielle) de ses habitants » (Estèbe, 2008).

La coexistence d'un "régime central ou métropolitain », et d'un " régime périurbain » (Estèbe, 2008) ne facilite pas la constitution d'un "Gargantua » métropolitain qui demeure toujours un objectif en filigrane des réformes territoriales passées et à venir ${ }^{4}$. Or, cette recherche incessante de la "surterritorialité ", par l'élargissement des périmètres et des compétences de l'intercommunalité centrale, est critiquée parce qu'elle fait « l'impasse sur l'indispensable gouvernance multi-niveaux en renforçant l'appétit pour le gouvernement unique » (Vanier, 2008).

Davantage au " goût du jour » (Jouve, 2004), la gouvernance correspond à la recherche du consensus et à la capacité de négocier. Selon Gilles Pinson, l'approche des politiques locales a substitué au "système politico-administratif local », la notion "gouvernance urbaine " qui permet de "subsumer les phénomènes de pluralisation des systèmes d'acteurs urbains » (Le Galès, 1995). Aujourd'hui, les "relations horizontales », c'est à dire « les conflits, coopérations et logiques d'alliance qui se nouent entre acteurs, groupes et organisations présents dans la ville peuvent être désormais considérées comme la première explication des formes que prend l'action publique urbaine " (Pinson, 2010). Même remarque par Philippe Subra qui démontre combien les interactions, les frictions ou les conflits entre acteurs publics ou privés sont devenus constitutifs de l'aménagement du territoire (Subra, 2007).

L'enjeu central de la gouvernance urbaine n'est plus tant pour les élus de décider unilatéralement mais "d'introduire assez de coopération entre les éléments épars qui constituent la communauté pour faire en sorte que les choses se fassent » (Stone, 1989). En 
effet, les situations de pluralisme rendent vain le recours au commandement hiérarchique comme mode de coordination (Pinson, 2010). La "complexification des sociétés urbaines" rend nécessaire le recours à des "innovations instrumentales " (Lascoumes, Le Gales, 2005). Selon Gilles Pinson (2002), l'innovation en matière d'action publique (partenariats, contrats, projets, pactes, chartes), l'accent mis sur les grands processus mobilisateurs (grands projets urbains, planifications stratégiques, grands évènements, etc.) sont autant d'éléments qui révèlent l'importance prise par la densification et la gestion des relations horizontales dans la gouvernance urbaine.

Le concept "d'interterritorialité ", lecture contemporaine des rapports entre pouvoir et territoire développée par Martin Vanier, s'inscrit directement dans les travaux sur la gouvernance: "si le gouvernement est la forme politique la plus aboutie du territoire, la gouvernance est certainement celle de l'interterritorialité » (Vanier, 2008). L'auteur part de l'hypothèse que " l'organisation politique des territoires est devant un nouveau devoir, celui de l'interterritorialité, qui la conduira à consacrer davantage d'efforts à ce qui se décide, se construit, se régule, se gère, entre territoires, qu'à l'intérieur de chacun d'eux pour luimême » (Vanier, 2008). Plusieurs processus sont interpellés par l'hypothèse interterritoriale comme la périurbanisation " qu'il s'agit de reconnaître comme un tiers espace, celui d'une interterritorialité au quotidien " ou la diversification sociale de nos rapports à l'espace, avec l'exemple de nos mobilités plus complexes.

Face à ces problématiques, il est devenu urgent de travailler à " l'organisation d'une action publique interterritoriale » (Vanier, 2008) par exemple avec la mise en place de "lieux institutionnels originaux réunissant autour d'une même table des acteurs des réseaux (transporteurs, autorités organisatrices) et des acteurs des territoires (élus locaux, gestionnaires d'équipements...) dans la diversité des échelles géographiques concernées » (Offner, 2002). Dès lors, il est question de savoir si les syndicats mixtes de transport promus en 2000 par la loi SRU, constituent une véritable « innovation institutionnelle en matière de coordination des politiques de transport» (Kaufmann, Pattaroni, 2010) susceptible de contribuer à l'émergence d'une « action publique interterritoriale ».

\section{2/ L'organisation des transports publics depuis les années 1970 face à la recherche du « bon » gouvernement urbain}

Avant de préciser les caractéristiques des nouvelles formes de syndicats mixtes, il convient de revenir synthétiquement sur la trajectoire des politiques locales de transport public tracée par Philippe Menerault (2005). L'objectif est de montrer que les formules de "gouvernance » ne sont pas nouvelles même si l'inclusion de la compétence transport dans des "gouvernements urbains" demeure une injonction forte des successives réformes territoriales.

Témoignage de la politique fédérative des années 1960-1970, la constitution des communautés urbaines dans les métropoles d'équilibre vise à doter les agglomérations d'institutions intégrées disposant de compétences étendues (Menerault, 2005). Les " transports urbains de voyageurs" compte parmi les compétences obligatoires retenues par la loi. Ce sont pourtant des syndicats mixtes ouverts qui vont jouer le rôle d'autorité organisatrice des transports urbains sur le périmètre de ces intercommunalités. Pourquoi ? D'abord parce que les transports publics sont perçus comme une compétence spécifique, dissociée de l'urbanisme, comme l'illustre à la même époque (début des années 1970), la création d'une ressource propre, le versement-transport. Ensuite, parce que le poids démographique des grandes agglomérations dans leur département, ainsi que le rôle de l'échelon départemental dans les transports publics de l'époque, militent en faveur d'une association du Conseil Général à l'organisation des déplacements urbains. Ainsi cette politique fédérative, tout en s'inscrivant dans une démarche forte d'intégration des compétences au sein d'un "gouvernement urbain », encourage les coopérations entre différents échelons d'AOT (urbain et interurbain) à travers des syndicats mixtes. 
Les communautés urbaines, marquées par une stabilité en terme de statut et de périmètre, sont maintenues au sommet de l'intégration intercommunale française par la loi Chevènement soit plus de 30 ans après leur création. Durant cette période, les autres démarches en faveur d'une intercommunalité plus forte n'ont pas été couronnées de succès : la volonté de généraliser les communautés urbaines (1968), la loi sur les fusions de communes (1971), le développement des districts urbains (dès 1959) ou la loi ATR (1992) avec les communautés de ville, n'aboutissent pas à l'émergence d'un gouvernement intégré dans les grandes agglomérations françaises.

En décalage avec cette difficile structuration intercommunale, les coopérations autour de la compétence "transport public" se développent selon une logique sectorielle sous l'impulsion du versement-transport (Menerault, 1993). Jusqu'en 1999, le nombre de districts ayant une compétence relative à l'organisation des transports publics, demeure relativement modeste (de 10 à $20 \%$ des AOTU) à côté de l'importance des formes syndicales (SIVU, SIVOM) qui comptent pour plus du tiers des AOTU.

En 1999, le retour du "gouvernement » est opéré par la loi Chevènement qui prône la " cohérence intersectorielle " en pointant la gestion sectorisée des compétences (notamment l'organisation des réseaux de transport public). En créant les communautés d'agglomération qui intègrent dans leur socle de compétence obligatoire « l'organisation des transports publics ", la loi Chevènement provoque une grande redistribution des statuts des AOTU : plus des 2/3 d'entre-elles changent de statuts entre 1999 et 2004, au détriment des SIVU et SIVOM et au profit des nouvelles communautés d'agglomération, devenues la première forme d'autorité organisatrice de transport urbain (Richer, 2005). Pourtant, l'important bouleversement des AOTU en faveur d'intercommunalités intégrées contraste avec une nouvelle dynamique en faveur des syndicats mixtes. Celle-ci contribue à alimenter la tension entre cohérence spatiale et intersectorielle (Offner, 2002) et à motiver notre regard sur les nouvelles formes de syndicats mixtes de transport.

\section{3/ Les syndicats mixtes de transport : d'une formule syndicale ancienne aux ambitions renouvelées de la loi SRU (2000)}

Les syndicats mixtes sont une forme de coopération intercommunale ancienne puisqu'elle a été créée par le décret-loi du 30 octobre $1935^{5}$. Le décret du 20 mai 1955 permet aux syndicats mixtes de faire coopérer des collectivités territoriales de niveaux différents, élargissant ainsi leur champ d'intervention. Les syndicats mixtes disposent de règles de fonctionnement souples, répondant aux besoins de coopérations flexibles (création et dissolution facilitées, coopération à durée déterminée ou indéterminée) et à la carte (adhésion libre), entre différentes collectivités sous deux formes :

- les syndicats mixtes «fermés » se composent soit de communes et d'établissements publics de coopération intercommunale (EPCI), soit uniquement d'EPCI. Des AOTU, communes ou $\mathrm{EPCl}$, peuvent donc constituer un syndicat mixte fermé et lui transférer leur compétence en matière d'organisation des transports urbains.

- Les syndicats mixtes "ouverts " classiques associent d'un commun accord des collectivités, institutions ou établissements publics; ceux qui relèvent de la SRU visent plus spécifiquement l'association d'autorités organisatrices de transport avec des compétences adaptées à la coordination des services qu'elles organisent. 
Tableau 1 : Les différentes formes de syndicats intercommunaux et syndicats mixtes (Données statistiques : CERTU-DGMT-GART-UTP)

\begin{tabular}{|c|c|c|c|c|}
\hline \multicolumn{2}{|c|}{$\begin{array}{l}\text { Syndicats intercommunaux, } \\
\text { regroupement de communes }\end{array}$} & \multicolumn{3}{|c|}{$\begin{array}{c}\text { Syndicats mixtes (SM), regroupement de différents } \\
\text { types de personne moral de droit public }\end{array}$} \\
\hline SIVU & SIVOM & SM fermé & \multicolumn{2}{|c|}{ SM ouvert } \\
\hline $\begin{array}{c}\text { Syndicat } \\
\text { intercommunal à } \\
\text { vocation unique }\end{array}$ & $\begin{array}{c}\text { Syndicat } \\
\text { intercommunal à } \\
\text { vocation multiple }\end{array}$ & $\begin{array}{l}\text { SM de «droit } \\
\text { commun » }\end{array}$ & $\begin{array}{l}\text { SM ouvert } \\
\text { " classique » }\end{array}$ & $\begin{array}{l}\text { SM de transport } \\
\text { de type SRU }\end{array}$ \\
\hline $\begin{array}{l}\text { Une seule } \\
\text { compétence qui } \\
\text { peut être relative } \\
\text { aux transports } \\
\text { urbains }\end{array}$ & $\begin{array}{l}\text { Plusieurs } \\
\text { compétences dont } \\
\text { une peut être } \\
\text { relative aux } \\
\text { transports urbains }\end{array}$ & $\begin{array}{l}\text { Composé de } \\
\text { commune(s) et/ou } \\
d^{\prime} E P C l . \text { Peut avoir } \\
\text { une compétence } \\
\text { relative aux } \\
\text { transports urbains }\end{array}$ & $\begin{array}{l}\text { Regroupe des } \\
\text { coll. territoriales et } \\
\text { d'autres } \\
\text { personnes } \\
\text { morales de droit } \\
\text { public. Peut avoir } \\
\text { une compétence } \\
\text { relative aux } \\
\text { transports urbains }\end{array}$ & $\begin{array}{l}\text { Composé } \\
\text { uniquement } \\
\text { d'AOT. } \\
\text { Compétences } \\
\text { relatives aux } \\
\text { transports urbains } \\
\text { et/ou non urbains } \\
\text { (Structure dédiée } \\
\text { aux transports) }\end{array}$ \\
\hline $\begin{array}{l}\text { De } 28 \% \text { des } \\
\text { AOTU en } 1999 \\
\text { (57 réseaux) à } \\
7 \% \text { en } 2008 \\
\text { (19 réseaux) }\end{array}$ & $\begin{array}{c}\text { De } 5 \% \text { des } \\
\text { AOTU en } 1999 \\
\text { (11 réseaux) à 1\% } \\
\text { en } 2008 \\
(3 \text { réseaux) }\end{array}$ & \multicolumn{2}{|c|}{$\begin{array}{c}\text { De } 5 \% \text { des AOTU en } 1999 \\
\text { (10 réseaux) à } 10 \% \text { des AOTU en } \\
2008 \text { ( } 27 \text { réseaux) }\end{array}$} & $\begin{array}{l}\text { Création en } \\
2000.7 \text { SM SRU } \\
\text { en } 2008 ; 11 \text { en } \\
2010 .\end{array}$ \\
\hline
\end{tabular}

Du point de vue des transports publics, les syndicats mixtes de la loi SRU correspondent, en quelque sorte, au « $3^{\text {ème }}$ âge » de la formule juridique :

- La première vague significative de créations de syndicats mixtes ayant une responsabilité en matière de transport remonte aux années 1960-1970: à Lille, Dunkerque, Lyon, Grenoble, Clermont-Ferrand ou Toulouse se sont créés, sous cette forme, des AOTU associant l'EPCI au Conseil Général. La stabilité de ces syndicats mixtes d'agglomérations de grande taille est devenue dernièrement plus fragile alors même que la loi SRU prône la création de syndicats associant différents niveaux d'AOT. La participation des Départements aux AOTU, déjà largement remise en cause dans plusieurs agglomérations ${ }^{6}$, sera rendue prochainement impossible par la loi de réforme des collectivités territoriales du 16 décembre $2010^{7}$.

- Depuis la loi Chevènement, un deuxième type original de syndicat mixte de transport est apparu, provoquant une progression sensible du nombre d'AOTU de cette forme (de 10 à 27 syndicats mixtes AOTU entre 1999 et 2008 dans un contexte général d'augmentation du nombre de réseaux). II s'agit de syndicats créés en réaction à la création de communautés d'agglomération, donc à la loi Chevènement. Ces syndicats mixtes "fermés » ou de " droit commun » organisent la compétence sur les transports publics urbains en lieu et place de leurs membres, principalement des communautés d'agglomération. Prolongeant le plus souvent une structure syndicale à vocation transport, ils répondent à l'objectif de préserver l'unité du réseau de transports publics urbains face à la création de communautés d'agglomération qui fragmentent les anciens périmètres de transports urbains (Richer, 2005).

Globalement, ces deux premières formes de syndicats mixtes ayant une responsabilité en matière de transport représentent 1 AOTU sur 10 en 2008. Loin d'être négligeables, ces formules sont souvent employées dans les réseaux de transports des aires urbaines les plus peuplées. II s'agit même du statut juridique le plus représenté dans les réseaux de transport urbain qui disposent d'un Transport Collectif en Site Propre (TCSP) en service ( $40 \%$ des AOTU).

- Les syndicats mixtes SRU constituent la troisième évolution notable du statut. La loi "Solidarité et Renouvellement Urbain» a offert la possibilité à des AOT de se regrouper au sein d'un nouveau type de structure afin de mieux coordonner leurs politiques relatives aux transports, qu'ils soient urbains et/ou non urbains. Ce syndicat 
mixte SRU est spécifiquement dédié aux transports et sa composition est réservée aux AOT contrairement aux autres types de syndicats mixtes. Sans se substituer à ses membres, un syndicat mixte SRU doit obligatoirement prendre en charge trois compétences : la coordination des services de transport; la mise en place d'une communauté tarifaire de titres uniques ou unifiés; l'organisation d'un système d'information multimodale à l'intention des usagers. L'objectif principal est de favoriser l'usage des transports collectifs quels qu'ils soient, en réduisant au maximum les obstacles que peut rencontrer un usager lors du passage d'un réseau de transport à un autre. Le syndicat peut aussi assurer des compétences optionnelles en lieu et place de ses membres, tel que l'organisation de services réguliers et de services à la demande ainsi que la réalisation et la gestion d'équipements et d'infrastructures de transport. Les syndicats mixtes SRU peuvent être dotés d'une ressource spécifique permettant en particulier le financement des missions d'intérêt commun, le Versement-Transport Additionnel (VTA).

Tableau 2 : Les trois âges des syndicats mixtes de transport

\begin{tabular}{|l|l|l|l|l|}
\hline Période & Politique & $\begin{array}{l}\text { forme de } \\
\text { syndicat mixte }\end{array}$ & $\begin{array}{l}\text { Composition } \\
\text { fréquente }\end{array}$ & Objectif \\
\hline $\begin{array}{l}\text { Depuis } \\
\text { les } \\
\text { années } \\
1960\end{array}$ & $\begin{array}{l}\text { Métropoles d'équilibre, } \\
\text { politique fédérative (loi } \\
\text { relative aux communautés } \\
\text { urbaines, 1966) }\end{array}$ & $\begin{array}{l}\text { SM ouvert } \\
\text { « classique » }\end{array}$ & $\begin{array}{l}\text { Communauté } \\
\text { Urbaine et } \\
\text { Département }\end{array}$ & $\begin{array}{l}\text { Organisation et/ou gestion } \\
\text { d'un réseau de transport } \\
\text { collectif dans les grandes } \\
\text { agglomérations. }\end{array}$ \\
\hline $\begin{array}{l}\text { Depuis } \\
1999\end{array}$ & $\begin{array}{l}\text { Renforcement des } \\
\text { intercommunalités et } \\
\text { cohérence intersectorielle } \\
\text { (loi Chevènement, 1999) }\end{array}$ & $\begin{array}{l}\text { SM fermé de } \\
\text { « droit } \\
\text { commun » }\end{array}$ & $\begin{array}{l}\text { Communauté(s) } \\
\text { d'agglomération(s) } \\
\text { et communes }\end{array}$ & $\begin{array}{l}\text { Maintien du PTU antérieur } \\
\text { pour pérenniser l'échelle } \\
\text { d'organisation du réseau } \\
\text { de TCU. }\end{array}$ \\
\hline $\begin{array}{l}\text { Depuis } \\
2000\end{array}$ & $\begin{array}{l}\text { Politique de transport et } \\
\text { cohérence spatiale (loi } \\
\text { SRU, 2000) }\end{array}$ & $\begin{array}{l}\text { SM ouvert de } \\
\text { type « SRU » }\end{array}$ & $\begin{array}{l}\text { Différentes AOT } \\
\text { (AOTU et } \\
\text { Département) }\end{array}$ & $\begin{array}{l}\text { Coordination des services, } \\
\text { politique tarifaire et } \\
\text { information multimodale. }\end{array}$ \\
\hline
\end{tabular}

Au-delà de l'ambition de la loi, la deuxième partie étudie plus précisément les modalités d'application locale des syndicats mixtes de transport SRU qui se sont formés avant 2008. 


\section{PARTIE 2. Etude des syndicats mixtes de transport de la loi SRU (2000- 2008) : un nouvel instrument de gouvernance interterritoriale?}

On dénombre en 2010, 11 syndicats mixtes de transport référencés comme conforme à la loi SRU par le Ministère des transports ${ }^{8}$, mais pour des raisons de temporalité de l'étude et de recul nécessaire à la mise en place des compétences, notre échantillon est composé des 7 premiers syndicats créés avant 2008.

Tableau 3 : Les syndicats mixtes SRU existants en 2008 intégrés à l'étude

\begin{tabular}{|c|c|c|c|c|c|}
\hline & $\begin{array}{l}\text { Nom complet (et } \\
\text { date de création) }\end{array}$ & $\begin{array}{l}\text { Autorités organisatrices de } \\
\text { transport adhérentes }\end{array}$ & $\begin{array}{l}\text { Echelle de } \\
\text { compétence } \\
\text { du syndicat }\end{array}$ & $\begin{array}{l}\text { Population } \\
2008 \text { du } \\
\text { périmètre }\end{array}$ & $\begin{array}{l}\text { Budget } 2008 \\
\text { et part du } \\
\text { fonctionnement }\end{array}$ \\
\hline SYMITAM & $\begin{array}{l}\text { Syndicat Mixte des } \\
\text { Transports des } \\
\text { Alpes-Maritimes } \\
(2005)\end{array}$ & $\begin{array}{l}1 \text { Conseil Général (Alpes- } \\
\text { Maritimes), } \\
3 \text { communautés } \\
\text { d'agglomération (Nice, Sophia } \\
\text { Antipolis, Riviera Française), } \\
3 \text { SIVU transport (Cannes, } \\
\text { VARMER, Grasses) }\end{array}$ & $\begin{array}{l}\text { Département } \\
\text { des Alpes- } \\
\text { Maritimes }\end{array}$ & $\begin{array}{l}1 \text { million } \\
\text { d'habitants } \\
\text { dont } 95 \% \\
\text { dans les } \\
\text { PTU }\end{array}$ & $\begin{array}{l}500000 € \\
\text { (93\% de fonct.) }\end{array}$ \\
\hline SMTCH & $\begin{array}{l}\text { Syndicat Mixte des } \\
\text { Transports en } \\
\text { Commun de } \\
\text { l'Hérault (2004) }\end{array}$ & $\begin{array}{l}1 \text { Conseil Général (Hérault), } \\
4 \text { communautés } \\
\text { d'agglomération (Montpellier } \\
\text { Agglomération, Béziers- } \\
\text { Méditerrannée, Bassin de } \\
\text { Thau, Hérault-Méditerrannée) }\end{array}$ & $\begin{array}{l}\text { Département } \\
\text { de l'Hérault }\end{array}$ & $\begin{array}{l}1 \text { million } \\
\text { d'habitants } \\
\text { dont } 60 \% \\
\text { dans les } \\
\text { PTU }\end{array}$ & $\begin{array}{l}62000000 € \\
\text { (99\% de fonct.) }\end{array}$ \\
\hline SMTCO & $\begin{array}{l}\text { Syndicat Mixte des } \\
\text { Transports } \\
\text { Collectifs de l'Oise } \\
(2006)\end{array}$ & $\begin{array}{l}1 \text { Conseil Régional (Picardie), } \\
1 \text { Conseil Général (Oise), } \\
3 \text { communautés } \\
\text { d'agglomération (Beauvaisis, } \\
\text { Creil, Compiègne), } \\
2 \text { communautés de communes } \\
\text { (Sablons, Pierre-Sud-Oise), } \\
6 \text { communes. }\end{array}$ & $\begin{array}{l}\text { Département } \\
\text { de l'Oise }\end{array}$ & $\begin{array}{l}800000 \\
\text { habitants } \\
\text { dont } 43 \% \\
\text { dans les } \\
\text { PTU }\end{array}$ & $\begin{array}{l}8500000 € \\
\text { (85\% de fonct.) }\end{array}$ \\
\hline SMCTCM & $\begin{array}{l}\text { Syndicat Mixte de la } \\
\text { Communauté } \\
\text { Tarifaire en } \\
\text { Charente-Maritime } \\
\text { (2001) }\end{array}$ & $\begin{array}{l}1 \text { Conseil Général (Charente- } \\
\text { Maritime); } \\
3 \text { communautés } \\
\text { d'agglomération (La Rochelle, } \\
\text { Pays Rochefortais, Royan } \\
\text { Atlantique); } \\
1 \text { SIVU-transport (Saintes) }\end{array}$ & $\begin{array}{l}\text { Département } \\
\text { de Charente- } \\
\text { Maritime }\end{array}$ & $\begin{array}{l}600000 \\
\text { habitants } \\
\text { dont } 50 \% \\
\text { dans les } \\
\text { PTU }\end{array}$ & $\begin{array}{l}700000 € \\
\text { (55\% de fonct.) }\end{array}$ \\
\hline SMTS & $\begin{array}{l}\text { Syndicat Mixte des } \\
\text { Transports } \\
\text { Suburbains de } \\
\text { Nancy (2002) }\end{array}$ & $\begin{array}{l}1 \text { Conseil Général (Meurthe et } \\
\text { Moselle), } \\
1 \text { communauté urbaine } \\
\text { (Nancy), } \\
1 \text { communauté de communes } \\
\text { (Pompey) }\end{array}$ & $\begin{array}{l}17 \text { communes } \\
\text { sur les } 41 \text { des } \\
\text { deux } \\
\text { communautés }\end{array}$ & $\begin{array}{l}221000 \\
\text { habitants }\end{array}$ & $\begin{array}{l}6180000 € \\
\text { (72\% de fonct.) }\end{array}$ \\
\hline SMITAM & $\begin{array}{l}\text { Syndicat Mixte pour } \\
\text { l'Intercommunalité } \\
\text { des Transports de } \\
\text { l'Agglomération } \\
\text { Mancelle (2004) }\end{array}$ & $\begin{array}{l}1 \text { communauté urbaine (Le } \\
\text { Mans), } \\
1 \text { communauté de communes } \\
\text { (l'Antonnière) }\end{array}$ & $\begin{array}{l}12 \text { communes } \\
\text { (intégralité des } \\
\text { deux } \\
\text { communautés) }\end{array}$ & $\begin{array}{l}200000 \\
\text { habitants }\end{array}$ & $\begin{array}{l}300000 € \\
\text { (96\% de fonct.) }\end{array}$ \\
\hline SMITEEB & $\begin{array}{l}\text { Syndicat Mixte des } \\
\text { Transports de l'Est } \\
\text { de l'Etang-de-Berre } \\
\text { (2001) }\end{array}$ & $\begin{array}{l}1 \text { Conseil Général (Bouches- } \\
\text { du-Rhône), } \\
1 \text { communauté urbaine } \\
\text { (Marseille), } \\
2 \text { communautés } \\
\text { d'agglomération (Pays d'Aix, } \\
\text { Berre-Salon-Durance) }\end{array}$ & $\begin{array}{l}8 \text { communes } \\
\text { de l'Est de } \\
\text { l'Etang de } \\
\text { Berre }\end{array}$ & $\begin{array}{l}140000 \\
\text { habitants }\end{array}$ & $\begin{array}{l}18600000 € \\
\text { (85\% de fonct.) }\end{array}$ \\
\hline
\end{tabular}

Ce retour d'expérience sur les syndicats mixtes SRU créés entre 2000 et 2008 a été construit à partir d'une approche croisant : les données recensées au cours d'entretiens semi-directifs auprès des responsables techniques des sept syndicats mixtes, les données mobilisables à partir des documents bibliographiques, l'exploitation des bases de données notamment les résultats de l'enquête TCU Certu-DGMT-GART-UTP 2007 et de I'URSSAF. L'analyse des syndicats mixtes SRU est orientée par une série de questionnement sur les motivations à l'origine de leur création, sur les périmètres de compétence, sur les jeux d'acteurs, sur les compétences et sur les ressources à disposition des syndicats. 


\section{1/ Quelles sont motivations à l'origine de la création des syndicats mixtes SRU ?}

La création d'un syndicat mixte SRU repose avant tout sur la volonté de collectivités locales de travailler ensemble à une meilleure intégration de leurs réseaux afin d'améliorer l'offre globale de transport public proposée à l'usager. Sur les sept cas d'étude, cette volonté préexistait antérieurement à la promulgation de la loi SRU en 2000 même s'il faut distinguer deux cas de figure :

- Cinq des sept syndicats mixtes SRU mis en place avant 2008 résultent de la transformation de structures partenariales pré-existantes qui étaient dédiées à la gestion de réseaux de transports locaux, a savoir: trois syndicats mixtes de droit commun (Charente Maritime, agglomération du Mans et Nancy Suburbain ${ }^{9}$ ), un syndicat intercommunal à vocation unique (le SMITEEB) et une société d'économie mixte détenue en majorité par le Conseil Général de l'Hérault (SMTCH). Dans ce cadre, la motivation à l'origine de la création d'un syndicat mixtes SRU est avant tout pragmatique : il s'agit de maintenir et de pérenniser les services existants à l'échelle de l'ancien périmètre.

- Le SMTCO de l'Oise et le SYMITAM ont été créés " ex nihilo " en qualité de syndicats mixtes SRU dans le cadre de politiques d'aménagement du territoire. Le premier participe à la mise en œuvre de l'Agenda 21 du Conseil Général de l'Oise ; le second est le fruit des réflexions menées suite au diagnostic des déplacements des ménages sur la bande côtière des Alpes-Maritimes. La motivation ici est davantage conforme aux ambitions de la loi puisque la nouvelle structure syndicale répond à une volonté de développer les transports collectifs et de réfléchir à une meilleure connexion des réseaux.

Ainsi, l'outil syndicat mixte SRU mis à disposition des collectivités en décembre 2000 a été principalement employé dans un premier temps pour modifier les structures juridiques existantes chargées de la gestion de services de transport. C'est seulement à partir de 2005 que la création des syndicats mixtes de l'Oise et des Alpes-Maritimes a validé une nouvelle démarche de coopération entre autorités organisatrices de transport.

\section{2/ Quelle est l'échelle des syndicats mixtes SRU et leurs périmètres de compétence?}

Le périmètre du syndicat mixte de transport SRU est fixé librement, d'un commun accord entre les AOT membres. Cette disposition s'est traduite par des périmètres de compétences de taille très variable s'adaptant à des réalités locales très différentes. Ainsi, les syndicats mixtes SRU existant en 2008 couvrent une échelle territoriale allant du simple regroupement de communes à l'intérieur de plusieurs PTU jusqu'au département. II n'y a pas, à cette date, de syndicat mixte SRU exerçant ses compétences à l'échelle régionale.

- Quatre des sept syndicats mixtes SRU ont pour périmètre de compétence le département : pour les syndicats mixtes de Charente-Maritime, des Alpes-Maritimes, de l'Hérault et de l'Oise, le Conseil Général joue un rôle important en cohérence avec le périmètre du syndicat qui couvre l'ensemble du territoire départemental.

- Les trois autres structures exercent leurs missions sur une échelle plus urbaine, autour de PTU contigus (Le Mans et Nancy) ou " à cheval " sur plusieurs PTU (Etang de Berre). Sur ce dernier cas, la loi SRU a permis au réseau de l'étang de Berre de perdurer à l'échelle des 8 communes alors que le périmètre d'un syndicat mixte de droit commun aurait recouvert l'intégralité du territoire des AOTU membres soit les 69 communes de la communauté urbaine de Marseille-Provence-Métropole et des communautés d'agglomération de Berre-Salon-Durance et du Pays d'Aix. 
Les périmètres de compétence des syndicats mixtes existant couvrent des populations allant de 140000 habitants pour le SMITEEB (bassin de vie de l'est de l'Etang de Berre inséré dans l'aire métropolitaine marseillaise) au million d'habitants pour les syndicats de l'Hérault et des Alpes Maritimes. Les syndicats mixtes de type "SRU » sont absents des grandes aires urbaines françaises alors que les syndicats mixtes « AOTU » y sont traditionnellement bien représentés.

\section{3/ Quelles sont les autorités organisatrices de transport impliquées ou associées aux jeux d'acteurs des syndicats mixtes SRU?}

Les syndicats mixtes mis en place avant 2008 ont été le résultat de démarches impulsées soit par les Conseils Généraux, soit par les AOTU à l'échelle de périmètres de transport urbain existants. Le nombre d'adhérents est très variable: il y a seulement deux intercommunalités associées au syndicat de l'agglomération mancelle mais plus d'une dizaine de collectivités de niveaux différents dans le syndicat mixte de l'Oise. Dans ce cas, certaines communes et communautés de communes ont même acquis la compétence d'autorité organisatrice de transport afin de pouvoir adhérer au SMTCO, conformément aux dispositions législatives.

Six des sept syndicats mixtes SRU étudiés associent le Conseil Général : quatre d'entre eux exercent leurs compétences sur un périmètre départemental (SMCTCM de Charente Maritime, SMTCH de l'Hérault, SMTCO de I'Oise et SYMITAM des Alpes Maritimes) en rassemblant l'ensemble des AOTU présentes dans le département. Pour les syndicats de l'Etang de Berre et de Nancy, le Conseil Général est associé même si le périmètre de compétence est à l'échelle infra-départementale. Seul le SMITAM, syndicat pour l'intermodalité des transports de l'agglomération Mancelle, ne compte pas l'autorité départementale parmi ses membres.

Dans le panel étudié, on ne recense qu'un seul Conseil Régional ayant adhéré à un syndicat mixte SRU : il s'agit du Conseil Régional de Picardie, membre du SMTCO de l'Oise. Les Régions qui se sont pourtant vues attribuer, par cette même loi SRU, des compétences nouvelles en matière d'organisation des transports, n'adhèrent pas à ces syndicats. Si les Régions ne sont pas membres à part entière des syndicats mixtes SRU existants, elle n'en demeure partenaires de ceux-ci (exemple de la Région Poitou-Charentes avec le SMCTCM ou de la Région Provence-Alpes-Côte d'Azur avec le SYMITAM). Elles sont amenées à collaborer avec les structures syndicales en fournissant un appui technique et de conseil sur les actions concernant le réseau TER, en participant aux réunions techniques sur la coordination de l'offre ou en s'associant au financement ponctuel ou régulier des projets du syndicat.

Les syndicats mixtes SRU s'appuient également sur d'autres acteurs non membres du syndicat. Les transporteurs des réseaux urbains sont des " partenaires naturels " de ces syndicats tandis que la SNCF est associée, par exemple, aux projets de mise en compatibilité des systèmes de billettique incluant le maillon TER

\section{4/ Quelles sont les compétences obligatoires et optionnelles assurées par les syndicats mixtes SRU et les actions mises en œuvre?}

Les sept syndicats mixtes SRU analysés en 2008 ont développé inégalement des actions relevant de leurs compétences obligatoires de coordination des réseaux, d'information multimodale et d'intégration tarifaire :

- La coordination des services de transport: la majorité des acteurs interrogés perçoivent la fonction de coordination des services de transport comme primordiale. Ils considèrent que ce type de syndicats doit contribuer prioritairement à faire 
travailler ensemble les AOT membres afin de faire converger et d'harmoniser les politiques de transport de chacune d'elles. Cette gouvernance commune s'opère au sein des instances mises en place dans les syndicats mixtes SRU : comités techniques composés de techniciens des AOT membres et chargés de la conception des projets, et comité syndical, organe de validation des décisions composé d'élus des AOT membres. La coordination des services de transport est souvent au stade de réflexions et non d'actions.

- L'information multimodale des usagers : la thématique « information à l'intention des usagers " prise en charge par les syndicats mixtes SRU revêt des formes multiples (dépliants, panneaux d'information aux arrêts, centrale de mobilité, bornes d'information multimodale, ...). En 2008, cinq des sept syndicats analysés fournissent a minima des informations sur les lignes et les horaires des réseaux de services publics réguliers locaux (départementaux voire régionaux) sur leur site internet ou via une centrale d'information téléphonique. Parmi ces cinq syndicats, trois travaillent actuellement à la conception d'un calculateur d'itinéraires qui permettra aux usagers de planifier leur trajet d'adresse à adresse. Le SMTCO de l'Oise a conféré également le rôle de centrale de covoiturage à la centrale d'information mise en place.

- L'intégration tarifaire entre réseaux : en 2008, cinq des sept syndicats mixtes SRU ont mis en place des titres intermodaux, permettant à l'usager de voyager sur différents réseaux de transport. Ainsi, en Charente Maritime, le SMCTCM a mis en place des titres de transports " unifiés " permettant d'accéder aux principaux sites touristiques locaux. Le SMTS de Nancy Suburbain et son exploitant proposent aux usagers la carte "Simplicités ", système sans contact, permettant de charger les titres de transports du réseau que les clients veulent utiliser. Le SMTCO de l'Oise se penche sur la question de la conception d'une carte à puce qui pourrait regrouper les titres de transport des différents réseaux des AOT membres du syndicat, y compris les titres intermodaux en cours de développement. Cette mission dévolue aux syndicats mixtes SRU soulève toutefois des questions importantes relatives à la répartition des recettes de ces titres entre l'ensemble des exploitants. Le syndicat peut être amené à jouer le rôle de chambre de compensation (c'est le cas du SMTS de Nancy Suburbain et du SMCTCM de Charente Maritime). Les syndicats ne disposant que d'un effectif réduit de personnel technique et administratif peuvent être amenés à externaliser cette fonction du fait de sa complexité et de sa lourdeur en terme de temps dédié (Exemple du SMTCO de l'Oise).

\section{Tableau 3 : Les premières actions mises en œuvre par les syndicats mixtes SRU}

\begin{tabular}{|c|c|c|c|}
\hline & Coordination des services & Information multimodale & $\begin{array}{l}\text { Tarification coordonnée et } \\
\text { titres intermodaux }\end{array}$ \\
\hline $\begin{array}{l}\text { SYMITAM } \\
\text { (Alpes- } \\
\text { maritimes) }\end{array}$ & $\begin{array}{l}\text { Participation à la démarche } \\
\text { d'interopérabilité des réseaux } \\
\text { pilotée par la Région (vers une } \\
\text { mise en compatibilité des } \\
\text { matériels de billettique des } \\
\text { différentes AOT) ; } \\
\text { Coordination des plannings } \\
\text { d'équipement billettique. }\end{array}$ & $\begin{array}{l}\text { Dépliant à destination des } \\
\text { usagers relatifs aux TC } \\
\text { organisés par les AOT } \\
\text { membres; } \\
\text { Mise en place d'un portail } \\
\text { internet www.symitam.fr } \\
\text { Plan des grandes lignes des } \\
\text { réseaux (avril 2009); } \\
\text { Vers la mise en place d'un } \\
\text { système d'information } \\
\text { multimodale. }\end{array}$ & $\begin{array}{l}\text { Etude sur la mise de différents } \\
\text { titres (abonnement annuel, } \\
\text { pass journée); } \\
\text { Mise en place d'un } 1^{\text {er }} \text { titre } \\
\text { unitaire intermodal « Azur » } \\
\text { donnant accès à } 2 \text { réseaux; } \\
\text { Vers la mise en service de } \\
\text { titres intermodaux et } \\
\text { multimodaux en } 2009 \text { et } 2010 .\end{array}$ \\
\hline $\begin{array}{l}\text { SMTCH } \\
\text { (Hérault) }\end{array}$ & $\begin{array}{l}\text { Réflexion commune en } \\
\text { matière de tarification } \\
\text { coordonnée et d'information } \\
\text { sur les réseaux; } \\
\text { Coordination des différentes } \\
\text { initiatives des AOTU. }\end{array}$ & $\begin{array}{l}\text { Vers la mise en place d'une } \\
\text { centrale d'information } \\
\text { multimodale via un site } \\
\text { internet www.herault- } \\
\text { transport.fr; } \\
\text { Mise en place d'un calculateur } \\
\text { d'itinéraires. }\end{array}$ & $\begin{array}{l}\text { Mise en place de titres } \\
\text { uniques de transport pour tout } \\
\text { déplacement sur les réseaux } \\
\text { départemental et urbain de } \\
\text { Montpellier (projet d'extension } \\
\text { sur Béziers); } \\
\text { Mise en œuvre d'un système } \\
\text { billettique. }\end{array}$ \\
\hline
\end{tabular}




\begin{tabular}{|c|c|c|c|}
\hline $\begin{array}{l}\text { SMTCO } \\
\text { (Oise) }\end{array}$ & $\begin{array}{l}\text { Réflexion commune sur la } \\
\text { mise en place d'outils } \\
\text { communs; } \\
\text { Coordination des différentes } \\
\text { initiatives des AOTU membres } \\
\text { sous l'angle de l'intermodalité. }\end{array}$ & $\begin{array}{l}\text { Mise en place d'une centrale } \\
\text { d'information multimodale via } \\
\text { une plateforme téléphonique } \\
\text { et un site internet avec toutes } \\
\text { les informations sur les } \\
\text { différents réseaux www.oise- } \\
\text { mobilité.fr ; } \\
\text { Projet de système de services } \\
\text { à la mobilité «SISMO» } \\
\text { (calculateur d'itinéraires) ; } \\
\text { Mise en place d'une centrale } \\
\text { de covoiturage } \\
\text { www.covoiturage-oise.fr. }\end{array}$ & $\begin{array}{l}\text { Projet de mise en place d'une } \\
\text { billettique intermodale } \\
\text { regroupant tous les titres de } \\
\text { transport des différents } \\
\text { réseaux }\end{array}$ \\
\hline $\begin{array}{l}\text { SMCTCM } \\
\text { (Charente- } \\
\text { Maritime) }\end{array}$ & $\begin{array}{l}\text { Réflexion sur le } \\
\text { développement de } \\
\text { l'intermodalité par la } \\
\text { coordination des réseaux par } \\
\text { une étude des } \\
\text { correspondances, fréquence } \\
\text { des dessertes, adéquation des } \\
\text { horaires, tarification. }\end{array}$ & $\begin{array}{l}\text { Mise en place d'un système } \\
\text { d'information voyageur (SIV) } \\
\text { par panneaux d'information } \\
\text { dans les pôles d'échanges, } \\
\text { site internet } \\
\text { www.passpartout17.org } \\
\text { intégrant un moteur de } \\
\text { recherche d'itinéraire } \\
\text { intermodal. }\end{array}$ & $\begin{array}{l}\text { Déploiement d'un équipement } \\
\text { billettique-monétique sur tous } \\
\text { les réseaux; } \\
\text { Mise en place de titres de } \\
\text { transport unifiés (Pass'Partout } \\
17 \text {; Pass'Rochelais, Pass'La } \\
\text { Rochelle-Rochefort) et } \\
\text { combiné (train+bus sur axe La } \\
\text { Rochelle-Rochefort en lien } \\
\text { avec Région) }\end{array}$ \\
\hline $\begin{array}{l}\text { SMTS } \\
\text { (Nancy) }\end{array}$ & $\begin{array}{l}\text { Réflexion commune sur le } \\
\text { développement d'un système } \\
\text { d'information multimodale et } \\
\text { d'un système billettique } \\
\text { harmonisé }\end{array}$ & $\begin{array}{l}\text { Projet de développement d'un } \\
\text { système d'information } \\
\text { multimodale intégrant } \\
\text { l'information TER via un site } \\
\text { internet avec module de } \\
\text { recherche d'itinéraires }\end{array}$ & $\begin{array}{l}\text { Mise en place d'une } \\
\text { tarification intégrée sur le } \\
\text { périmètre syndical ; } \\
\text { Mise en place d'un nouveau } \\
\text { système billettique. }\end{array}$ \\
\hline $\begin{array}{l}\text { SMITAM } \\
\text { (Le Mans) }\end{array}$ & $\begin{array}{l}\text { Réflexion commune sur la } \\
\text { mise en place d'un titre unique }\end{array}$ & & $\begin{array}{l}\text { Mise en place d'un titre unique } \\
\text { de transport pour } \\
\text { l'intermodalité tramway/bus } \\
\text { (objectif 2011) }\end{array}$ \\
\hline $\begin{array}{l}\text { SMITEEB } \\
\text { (Etang de } \\
\text { Berre) }\end{array}$ & $\begin{array}{l}\text { Réflexion commune pour } \\
\text { mettre en œuvre une politique } \\
\text { coordonnée des transports } \\
\text { urbains et interurbains sur le } \\
\text { périmètre desservie }\end{array}$ & $\begin{array}{l}\text { Mise en place d'un site } \\
\text { internet www.bus-de-letang.fr } \\
\text { avec plan interactif des lignes }\end{array}$ & $\begin{array}{l}\text { Système billettique unique sur } \\
\text { le département (carte à puce) } \\
\text { tarification combinée avec la } \\
\text { SNCF ; Correspondances } \\
\text { gratuites avec les transports } \\
\text { départementaux. }\end{array}$ \\
\hline
\end{tabular}

Parmi les sept syndicats, quatre d'entre-eux (SMITAM Le Mans, SMITEEB Etang de Berre, SMTCH Hérault et SMTS Nancy) ont pris en charge la compétence optionnelle d'organisation de services réguliers de transports collectifs et plus rarement de services de transport à la demande (TAD). II s'agit soit de l'organisation de lignes régulières et de services de TAD entre PTU contigus ou du transfert de compétences du Département et des agglomérations en matière de transport scolaire ou de transport collectif urbain. Le réseau le plus important est celui géré par le SMTCH de l'Hérault avec 68 lignes régulières. Pour le syndicat de l'Hérault, l'enjeu principal de la coopération est la « libre circulation » de tous les scolaires sur le territoire du Département. Le périmètre du SMITEEB (Etang de Berre) est irrigué par 25 lignes régulières assurant les liaisons entre 8 communes des PTU de Marseille, d'Aix et de Salon-de-Provence. Le réseau mis en place par le SMTS (Nancy suburbain) pour assurer les liaisons entre les PTU de Nancy et de Pompey est composé de 3 lignes régulières. Enfin, le réseau SMITAM (Le Mans) est le plus petit réseau parmi les syndicats SRU existant puisqu'il ne comprend qu'une seule ligne de bus assurant huit rotations par jour entre l'Antonnière et le Mans.

La prise de compétence optionnelle relative à l'organisation de services de transports par les syndicats mixtes SRU assure la poursuite et le développement de l'offre de transport préexistante. Les quatre syndicats mixtes SRU ayant pris une compétence facultative consacrent la majorité de leur budget de fonctionnement à la mise en œuvre de cette mission : 93\% du budget de fonctionnement du SMTCH (Hérault) est dédié aux contrats passés avec les transporteurs tandis que les charges d'exploitation du SMITEEB s'élèvent à plus de $75 \%$ de son budget de fonctionnement. L'organisation de services de transport par un syndicat mixte SRU conditionne fortement leur marge de manœuvre financière. 


\section{5/ Quels sont les moyens humains et financiers à disposition des syndicats mixtes SRU pour mener leurs actions?}

La majorité des syndicats mixtes SRU enquêtés disposent de leurs propres ressources humaines mais en nombre réduit, de deux à six personnes. Seuls le SMITEEB (Etang de Berre) et le SMTCH (Hérault) s'appuient sur des équipes plus conséquentes (respectivement 24 et 48 personnes). Ce personnel est employé directement par le syndicat sauf dans le cas du SMITAM et du SMTS où il est mis à disposition et hébergés par une AOT membre. Les syndicats mixtes SRU font parfois appel à l'expertise de leurs AOT membres. Ainsi, le SYMITAM (Alpes Maritimes) sollicite fréquemment les services juridiques de ses AOT membres, ne possédant pas cette compétence juridique en interne.

Pour réaliser leurs missions, les syndicats mixtes SRU s'appuient principalement sur deux types de ressources financières, les contributions de leurs membres et le versement transport additionnel (VTA), dont le choix résulte d'une volonté politique :

- Deux syndicats mixtes SRU ont prévu dans leurs statuts à la fois de prélever un VTA et de faire participer financièrement leurs membres. II s'agit du SMTCH de l'Hérault et du SMITAM du Mans (seulement en cas de déficit d'exploitation de la ligne de bus prise en charge).

- Quatre syndicats mixtes SRU s'appuient sur les contributions de leurs membres mais ne prélèvent pas de VTA pour des raisons diverses : le SMCTCM (CharenteMaritime) n'a pas choisi d'instaurer un VTA depuis sa création en raison de l'existence d'un excédent annuel de fonctionnement ; le SMITEEB (Etang de Berre) n'a pas la possibilité d'instaurer un VTA du fait de la valeur des taux de VT en vigueur sur chaque PTU ; le SYMITAM des Alpes Maritimes ne projette pas d'instaurer un VTA considérant que la réalisation de ses missions actuelles ne nécessite pas d'apport financier complémentaire ; et le SMTS de Nancy n'a pas pu mettre en place un VTA pour l'instant en raison d'oppositions fortes des entrepreneurs locaux.

- Seul le SMTCO de l'Oise a choisi de prélever un VTA sans demander de contribution à ses membres. Dans les faits, le SMITAM du Mans est également dans le même cas de figure puisqu'en 2008, aucune contribution des membres n'a été effective, celle-ci n'étant déclenchée qu'en cas de déficit.

Outre les contributions des AOT membres du syndicat et/ou le prélèvement d'un VTA, certains syndicats mixtes SRU bénéficient de subventions accordées par des partenaires institutionnels externes. Ces subventions sont très souvent ponctuelles mais peuvent revêtir un caractère périodique: la Région Poitou-Charentes octroie une subvention annuelle au SMCTCM (Charente Maritime) qui représente $8 \%$ du budget de fonctionnement en 2008, pour développer l'intermodalité avec le réseau TER. 


\section{PARTIE 3. Questions vives pour la gouvernance des mobilités à travers l'étude des syndicats mixtes de transport de la loi SRU}

\section{1/ Un nouvel acteur dans le champ des transports publics: les syndicats mixtes SRU, « du vieux vin dans de nouvelles bouteilles »?}

Le développement des syndicats mixtes, certes encore modeste, participe à la multiplication des acteurs prenant part au transport public ${ }^{10}$. Cette pluralisation des intervenants pose question: y a-t-il trop d'acteurs dans la sphère des transports publics ? La création de nouveaux syndicats mixtes de transport est-elle vraiment nécessaire pour parvenir à des collaborations plus intenses entre AOT ? A en croire certaines expériences, comme celle de la région Alsace qui organise depuis longtemps un comité de coordination des AOT ayant finalement les mêmes fonctions qu'un syndicat mixte SRU, on peut en douter. Cependant, les contextes et situations locales ne sont pas transposables. Martin Vanier (2000) préfère assurer que " c'est au contraire la multiplication des échelles et des cadres territoriaux et, surtout, leur articulation qui représente la véritable innovation institutionnelle » comme les syndicats SRU tentent de l'orchestrer. Si le constat de la complexification de l'organisation territoriale ne fait aucun doute, "l'identification à réaliser doit intégrer une multiplicité de paramètres et de périmètres, à considérer en interface de façon plus ou moins systématique " (Nonn, 1996). La multiplicité des intervenants dans le domaine des déplacements n'est-il pas aussi une forme de reconnaissance de pratiques de mobilités plus complexes et si mal contenues dans des « bassins » fussent-ils de plus en plus larges (Vanier, 2008) ?

En outre, il convient également de s'interroger sur le caractère réellement "nouveau " et " innovant » de ces syndicats mixtes de transport dit "SRU ». II a déjà été évoqué le déclin (dont la loi SRU est en partie responsable) des premières formes de syndicats mixtes de transports qui ont associé depuis les années 1960, le conseil général à une $E P C I$ de grande taille comme par exemple à Lille ${ }^{11}$. II y a lieu de se demander si la loi SRU ne réinvente pas sous des modalités différentes des formes de coopération qui ont perduré pendant des décennies. En outre, le caractère novateur des syndicats mixtes peut être aussi questionné par rapport au rôle joué par une collectivité historique, en l'occurrence le Département. Dans la géopolitique des syndicats mixtes SRU, la position du Conseil Général est centrale : en réinventant son rôle dans la mosaïque territoriale des transports publics, l'acteur départemental apparaît dans un rôle "d'intercesseur territorial » (Behar, 2002). Pour les conseils généraux, la création d'une structure syndicale peut-être perçu comme une opportunité de faire valoir une situation privilégiée "d'AOT du périurbain » et leur légitimité sur la compétence " transport scolaire » (Richer, Menerault et al., 2007). Entre de jeunes AOTU communautaires, largement transformées suite à loi Chevènement, et un acteur régional «jeune » (Mancebo, 2003) à la compétence "transport d'intérêt régional » fraichement acquise, le département fait office de chef de file.

Du côté régional, il semble que l'on "partage peu volontiers ce qu'on vient de conquérir » (Vanier, 2008) ; ce qui explique une forme d'attentisme des Conseils Régionaux face à la création des syndicats mixtes SRU et au partage d'une compétence transport à " forte valeur politique » (Ollivier-Trigalo, 2007). Au-delà va se poser la question du positionnement de l'acteur régional face au développement de syndicats mixtes SRU à des échelles diverses et avec des modalités de fonctionnement variable (Hasiak, Jouve, 2010b). Si plusieurs syndicats se créent dans le périmètre régional, le Conseil Régional doit-il rester indifférent, doit-il participer individuellement à au financement de chacun d'eux, ou doit-il convertir les initiatives par la création d'un syndicat mixtes SRU à l'échelle régionale?

Au niveau des structures intercommunales, Caroline Gallez (2010) explique que « la solution des syndicats mixtes préconisée par la loi SRU, a priori séduisante, élude la question de l'implication des communes ou des collectivités périphériques en matière de transports collectifs". Certains syndicats légitiment même " une position attentiste des communes 
périphériques vis à vis d'une structure dont elles espèrent avant tout l'amélioration des conditions d'accessibilité au centre »(Gallez, 2010). En d'autres termes, l'adhésion à un syndicat mixte peut s'avérer pratique pour les communes et collectivités qui veulent profiter d'un accès au réseau de transport public urbain (ou à des services qui s'y rattachent) tout en désirant conserver une autonomie institutionnelle vis-à-vis de l'EPCI centrale. Ainsi, dans certaines circonstances, les syndicats mixtes SRU peuvent apparaître, par analogie avec l'analyse de Martin Vanier sur la contractualisation, moins comme «l'amorce de l'interterritorialité, que l'homéopathie qui permet de maintenir tant bien que mal le système des pseudo-souverainetés territoriales locales » (Vanier, 2008).

\section{2/ Réalité et virtualité des périmètres : le brouillage des limites d'organisation des transports publics urbains et non urbains}

D'un point de vue spatial, les syndicats mixtes SRU sont des acteurs difficilement définissables étant donnée la variété de leurs formes spatiales : d'un côté leur périmètre peut-être plus petit que les communautés intercommunalités adhérentes et d'un autre côté, leur territoire peut englober de nombreuses collectivités à l'échelle départementale et bientôt régionale. Cette souplesse maximum permet de dissocier le territoire des membres adhérents du périmètre de compétence du syndicat.

Les syndicats mixtes SRU partagent l'ambition d'effacer les frontières des réseaux de transport public et de faire évoluer le schéma traditionnel d'organisation des AOT strictement dual entre l'urbain (PTU avec VT) et le non-urbain (hors PTU sans VT). Sous l'effet des évolutions récentes, cette distinction a tendance à se fragmenter au profit d'un système aux composantes non encore stabilisées. Dans certains cas, elle laisse place à des seuils plus ou moins durables: il existe des PTU avec différents taux de VT et des zones hors PTU avec ou sans VTA (selon qu'elle appartiennent ou non à un espace à dominante urbaine). Dans le syndicat mixte des transports de l'Oise polarisé en grande partie par la région parisienne, 526 communes sont soumises à une modulation importante des taux de VT et/ou de VTA tandis que 117 y échappent. En effet, le périmètre de compétence du syndicat mixte (défini par ses membres) est différent du périmètre des ressources (défini selon les critères statistiques d'espace à dominante urbaine). Cet espace sur lequel est prélevé le VTA est d'ailleurs appelé «PTU virtuel », sous entendu mouvant en fonction des seuils statistiques et sans intensité politique, par opposition à un PTU bien réel, celui des institutions qui gèrent les transports publics urbains.

Les syndicats mixtes SRU tentent de traduire en terme de seuils, la fluidité toujours accrue de la dimension urbaine. Loin d'effacer complètement la limite entre l'intérieur et l'extérieur des PTU, ils introduisent même de nouveaux déséquilibres : une entreprise d'une commune incluse dans un syndicat mixte SRU bénéficiant des mêmes services peut-être taxée différemment selon que sa commune entre ou non dans un cadre statistique arbitraire. Ainsi, au-delà de la référence critiquable aux aires urbaines (Vanier, 2008; Gallez, 2010), la gouvernance des syndicats mixtes SRU joue aussi le jeu de la «différentiation territoriale » (Jouve, 2004). Alors que l'approche de l'intercommunalité « n'a pas intégré l'idée de l'abolition de la frontière entre ville et campagne dans les nouvelles formes de fonctionnement de la ville " (Wiel, 1994), les syndicats mixtes de transport SRU jouent un rôle ambigüe dans l'effacement de cette limite ${ }^{12}$. Pourtant, la question du sens de la distinction "urbain/non urbain » dans le domaine des transports publics doit être ouvertement posée.

\section{3/ Des compétences élargies à la gestion des services de mobilité mais une contribution encore modeste aux politiques intermodales}

D'un point de vue sémantique, beaucoup de spécialistes relèvent la transition de la thématique du "transport », à celle des "déplacements ", puis aujourd'hui à celle de la 
« mobilité » (Castel, 2007). Ainsi, les autorités en charge des transports publics ne sont plus seulement confrontées à la circulation des matériels ou au développement des infrastructures, elles doivent également répondre à la demande de service d'accompagnement de la mobilité. Les politiques publics de transport doivent, en quelque sorte, assurer "l'intégration servicielle des transports " (c'est-à-dire le développement de services d'assistance, de confort, d'agrément, mais également d'information multimodale, cartographique, en temps réel...), en somme favoriser le "Soft » d'un système de mobilité à côté du "Hard" de la technique de transport (Amar, 2004). Les syndicats mixtes SRU valident institutionnellement cette "mutation des transports urbains " (Amar, 2006) puisque ces structures n'ont pas vocation à être gestionnaires d'un réseau de transport mais plutôt d'orchestrer des services à la mobilité (information et tarification multimodale, covoiturage, transport à la demande...).

Cependant, la réalité des syndicats mixtes SRU ne va pas totalement dans ce sens : certains syndicats mixtes prennent une certaine liberté pour faire évoluer leurs prérogatives vers l'organisation de services réguliers de transport public. D'ailleurs, quelques structures sont davantage préoccupées par la gestion des lignes de transport que par les politiques intermodales prévues comme prioritaires. La lourdeur et la complexité de cette mission font passer au second plan la mise en œuvre des compétences obligatoires, du moins dans les premières années de fonctionnement du syndicat. Dès lors, la création de certains syndicats mixtes ne marque pas une réelle adhésion aux objectifs de la loi SRU mais relève plutôt d'une opportunité : celle de maintenir les services de transports sans se heurter à une reconfiguration profonde des périmètres d'AOTU, à l'image des motivations à l'origine de la constitution de nombreux syndicats mixtes «AOTU » après la loi Chevènement.

Le renforcement des compétences relatives à l'intermodalité des syndicats mixtes dépendra fortement des ressources financières du syndicat. Même si les contributions des membres devaient se maintenir, la question des ressources va se poser. L'instauration du versement transport additionnel n'est pas toujours bien accepté par les entrepreneurs et n'apparaît pas comme un " artifice » efficace pour trouver des ressources supplémentaires. Pour les rares syndicats mixtes SRU ayant déjà instauré un VTA, les acteurs interrogés dans le cadre de la présente étude relèvent la fragilité de l'assiette du VTA. Le VTA compte pour seulement $5 \%$ du budget du syndicat mixte SRU de l'Hérault. En outre, l'impossibilité de prélever la taxe additionnelle sur l'ensemble du territoire du syndicat mixte, les recettes limitées et la répartition complexe des taux expliquent la faible adhésion à la nouvelle formule de versement-transport.

Au final, on peut surtout soulever un décalage entre, d'une part, de puissants enjeux d'organisation des mobilités et de nombreux espoirs fondés par la littérature dans la gouvernance "interterritoriale » et, d'autre part, la modestie des nouvelles enveloppes institutionnelles en terme de moyens ou de compétences. D'ailleurs, il est difficile de connaître véritablement les marges de manœuvre des syndicats mixtes SRU. Quel est leur réel pouvoir? Rares sont les projets, mis à part peut-être l'information multimodale, que les syndicats mixtes peuvent conduire de l'amont jusqu'à l'aval, de la planification jusqu'à la réalisation. Leur fragile rôle de "maître d'ouvrage" doit être combiné à une maitrise d'œuvre au sein des AOT étant donnée la faiblesse des moyens humains.

Dans le domaine des transports publics, malgré ces escarmouches interterritoriales, il semble que l'on reste focalisé sur les structures de "gouvernement ». Est-ce que le rôle particulier joué par les transports publics « dans la territorialisation de l'action publique » (Gallez, 2010) qui limitent leur capacité à être intégré dans des dispositifs interterritoriaux ? Y a-t-il une crainte que ces structures à géométrie variable viennent brouiller le message qui vise à montrer les liens étroits entre un réseau de transports et son espace politique ${ }^{13}$ ? 


\section{CONCLUSION : les syndicats mixtes de transport SRU, apprentissage de la gouvernance ou ruse interterritoriale?}

Cet article a dressé un panorama des questions soulevées par la constitution récente de 7 syndicats mixtes SRU. Cette analyse transversale a le mérite de mettre en lumière des enjeux clés de l'organisation de la gouvernance des transports publics. Cependant, on peut lui reprocher d'être encore prématurée (ces nouvelles structures sont encore dans une phase de maturation et de légitimation), et de ne pas être actualisée étant donnée l'actualité riche qui anime cette forme de gouvernance: depuis 2008, les projets qui ont vu le jour pourraient nous amener à reconsidérer certaines interprétations, notamment par rapport à la place du Conseil Régional, moteur cette fois-ci, dans la création d'un Syndicat Mixte Intermodal Régional des Transports (SMIRT) dans le Nord-Pas-de-Calais.

Malgré les nombreuses croyances en la coopération des politiques publiques, le dispositif législatif en vigueur n'a pas tranché si bien que la phase actuelle peut encore apparaître comme transitoire (Estèbe, 2008). Les lois Chevènement et SRU cumulent l'enjeu de combiner négociation et arbitrage à travers un système politique qui n'impose pas l'unification des compétences (Wiel, 2010) : la loi Chevènement renforce le pouvoir urbain du groupement intercommunal central tandis que la loi SRU s'appuie sur un partenariat entre les institutions des espaces urbains et périurbains. Dans ces lois, on penche d'un côté pour une plus grande capacité d'arbitrage (plus de gouvernement), et de l'autre pour un accord de fond sur le projet (plus de gouvernance). La première formule peut induire des " conditions empêchant la recherche du consensus » (Wiel, 2010) et donc encourager des démarches mono-modales, tandis que la seconde implique des démarches plus consensuelles mais qui contournent les débats conflictuels?

Dans ce débat, les syndicats mixtes de transports de la loi SRU apparaissent comme des outils, dont l'application demeure perfectible, pour une gouvernance des réseaux de transport et des mobilités (lignes inter-PTU, articulation de l'offre urbaine et interurbaine, information multimodale, tarification combinée...). Mais pour forcer un peu le trait, on pourrait dire que rien ne ressemble moins à un syndicat mixte SRU qu'un autre syndicat mixte SRU. Quels points communs y a-t-il entre celui du Mans, gérant une ligne inter-PTU sur quelques communes, et le syndicat de l'Oise qui associe 13 AOT sur le périmètre départemental et qui prélève le VTA ? Du point de vue des échelles d'application, la loi a laissé une liberté totale aux coopérations syndicales : le périmètre d'intervention d'un syndicat mixte peut être différent de celui des autorités organisatrices qui le compose et, presqu'à coup sûr, sera en décalage avec la zone de perception du versement transport additionnel. La confusion peut être totale, mais le pragmatisme l'emporte. Le périmètre s'adapte aux paramètres locaux : du besoin ponctuel de coopération entre communes pour une ligne de bus qui sort du PTU à la politique intermodale à l'échelle d'un département ambitieux, les syndicats mixtes SRU sont modulables à souhait.

Le dénominateur commun devrait être le bloc des trois compétences obligatoires autour de l'articulation des services des AOT membres. Las! Le pragmatisme demeure aussi la règle et certains syndicats, motivés avant tout par l'organisation d'une offre de transport inter-PTU, font des compétences obligatoires des préoccupations secondaires. La compétence optionnelle est alors investie prioritairement si bien que le syndicat mixte SRU ressemble parfois plus à une AOTU classique qu'à un outil pour la gouvernance de l'intermodalité.

Si le syndicat mixte SRU perd de sa substance par l'adaptation de son périmètre et de ses compétences à une grande variété de contextes locaux, cette formule syndicale n'en demeure pas moins un « rouage essentiel à l'architecture interterritoriale » : Dans une forme "baroque ", " rustique » qui permet des alliances entre territoires de différentes natures et de différentes échelles, les syndicats mixtes SRU peuvent s'avérer être "une clé du système » selon les mots de Martin Vanier (2008). Ce que Jean-Marc Offner relève pour les 
procédures de planification des déplacements peut aussi valoir pour les syndicats mixtes SRU : leur efficacité réside moins dans leur contenu intrinsèque que dans leur aptitude à procurer des ingrédients pour les futurs politiques et décisions locales (Offner, 2003). La " vertu » des syndicats mixtes SRU n'est-elle pas d'élaborer « un processus d'apprentissage collectif » selon une expression employée toujours à propos des PDU (Frère, Menerault, Roussel, 2000) ? Ils peuvent participer à l'évolution des « référentiels » et à la transformation des systèmes d'action au profit d'une éventuelle action publique interterritoriale.

Cependant, la puissance des réseaux techniques exige que l'intercommunalité syndicale qui les gouverne, se transforme en objet politique (Vanier, 2008). En effet, la basse intensité politique des syndicats mixtes transforme "l'interterritorialité en une démarche technique d'entente gestionnaire " (Vanier, 2008), particulièrement palpable dans les acronymes utilisés (SMCTCM, SMTCH, SMTCO, SYMITAM,...). Cette situation contribue à alimenter l'idée que les syndicats mixtes sont des " ruses interterritoriales », particulièrement opaques, méconnues des usagers et peu légitimes étant donné leur éloignement de la représentation citoyenne. Difficile dans cette situation de parler d'amélioration de la gouvernance.

Si la maturation politique de ces formules syndicales pourrait renforcer leur capacité à construire une action publique interterritoriale, la question de leur pérennité doit être posée. Ces structures sont fragiles, non seulement par rapport à leur moyen financier et humain, mais aussi par rapport aux choix politiques qui peuvent être opérés. En particulier, la nouvelle loi de réforme des collectivités territoriales du 16 décembre 2010, issue des travaux du Comité "Balladur ", va " modifier structurellement l'architecture institutionnelle locale » en recyclant des "présupposés" (Behar, Estèbe, Vanier, 2009). La réforme privilégie des assemblages plus larges et plus intégrées (création de "Métropoles ») en brandissant de nouveau le mythe du "bon gouvernement métropolitain intégré ». Etant donnée la volonté explicite de "réduire le nombre de structures syndicales ", la nouvelle loi va entrer en tension avec les dispositifs de coopérations entre AOT qui peinent à se mettre en place, notamment dans les grandes aires métropolitaines. Récemment Daniel Behar a dessiné les contours d'un "âge III des pouvoirs locaux" qui prendrait en compte les enjeux interterritoriaux en généralisant " des formes de coopération à géométrie variable, adaptées à la diversité des enjeux à traiter » (Behar, 2010). L'application de la réforme des collectivités territoriales du 16 décembre 2010, risque de rendre ce nouvel âge encore bien illusoire. 


\section{BIBLIOGRAPHIE}

Amar G. (2004), Mobilités urbaines. Éloge de la diversité et devoir d'invention. Paris, Édition de l'Aube. Collection Société et Territoire. Série Prospective du présent. 251 p.

Amar G. (2006), « La transmodalité : une mutation des transports urbains », RATP, 12 p.

Behar D. (2002), "Le département : intercesseur territorial ? », INTER-REGIONS, la revue des agences de développement et des comités d'expansion, $n^{\circ} 240$, pp. 2-7

Behar D. (2007), «Après l'intercommunalité, l'intercommunautaire ? ", Pouvoirs Locaux n73, pp. $15-20$.

Béhar D., Estèbe Ph., Vanier M. (2009), " Mécano territorial : de l'ordre territorial à l'efficacité interterritoriale ", Pouvoirs Locaux N 83 IV/2009, pp. 79-83

Béhar D. (2010), « Pouvoirs locaux : vers un âge III », Note en ligne sur urbanisme.u-pec.fr/

Borraz O., John P. (2004), "The tranformation of urban political leadership in Wersten Europe ", International Journal of Urban and Regional Research, vol. 28, n¹.

Castel J.-C. (2007), Quand la mobilité oblige à repenser l'urbanisme, CERTU ?, $16 \mathrm{p}$.

Castells M. (2001), La Société en réseaux, l'ère de l'information, Fayard, pp. 473-530.

Dos Santos Guerrinha C. (2008), La gouvernance des régions urbaines. L'exemple des politiques de déplacements à Grenoble et Toulouse, Thèse de doctorat, Institut d'urbanisme de Paris (Université Paris 12), p.

Estèbe Ph. (2008), Gouverner la ville mobile. Intercommunalité et démocratie locale, coll. La Ville en Débats, PUF, $76 \mathrm{p}$.

Frère S., Menerault Ph., Roussel I. (2000), «PDU et dynamique des institutions à Lille et Valenciennes ", RTS n69, pp. 22-34

Gallez C. (2010), "Les politiques de transports publics en France face à l'impératif de cohérence territoriale », pp. 201-221, in Maksim H., Vincent S., Gallez C., Kaufmann V., L'action publique face à la mobilité, Ed. L'Harmattan, coll. Logiques sociales, 256 p.

Gallez C., Menerault Ph. (Dir.) (2008), Recomposition intercommunale et enjeux des transports publics en milieu urbain, Rapport de convention n ${ }^{\circ}$ C03-13 INRETS/ADEME, 277 p.

Gonin P. (1996), « Pays et développement local : l'état de la question dans la région Nord-Pasde-Calais », Hommes et Terres du Nord n², pp.113-121.

Hasiak S., Jouve N. (2010a), Les syndicats mixtes SRU : cadre juridique et mise en œuvre sur les territoires, CETE Nord-Picardie, Rapport d'étude pour la DREAL Picardie, $88 \mathrm{p}$.

Hasiak S., Jouve N. (2010b), Eléments de réflexion sur le développement de syndicats mixtes SRU en Picardie, CETE Nord-Picardie, Rapport d'étude pour la DREAL Picardie, $61 \mathrm{p}$.

Jemelin C., Vodoz L., Pfister B. (2004), «Entre accélération et rupture d'équilibre : une société à deux vitesses ", in Vodoz L., Pfister B., Jemelin C., Les Territoires de la mobilité. L'aire du temps, Presses polytechniques et universitaires romandes, pp. 379-383

Jouve B. (2004), "Gouvernance et décentralisation: les masques tombent enfin », EspacesTemps.net, Textuel, 17.01.2004 : http://espacestemps.net/document522.html

Kaufmann V., Pattaroni L. (2010), « Analyse des politiques de transport en France : un état des lieux de la recherche », pp. 23-50, in Maksim H., Vincent S., Gallez C., Kaufmann V., L'action publique face à la mobilité, Ed. L'Harmattan, coll. Logiques sociales, $256 \mathrm{p}$.

Lascoumes P., Le Galès P. (2005), " De l'innovation instrumentale à la recomposition de l'Etat », in Lascoumes P., Le Galès P., Gouverner par les instruments, Ed. Presses de Sciences Po., $370 \mathrm{p}$.

Le Galès P. (1995), « Du gouvernement des villes à la gouvernance urbaine », Revue française de science politique, vol.45, $\mathrm{n}^{\circ} 1$.

Maksim H., Vincent S., Gallez C., Kaufmann V. (2010), L'action publique face à la mobilité, Ed. L'Harmattan, coll. Logiques sociales, $256 \mathrm{p}$. 
Mancebo F. (2003), « Interterritorialités et nouvelles territorialités : quand l'enfer est pavé de bonnes intentions ", Recomposition territoriales et TIC, Caisse des Dépôts et Consignations, Castres, pp. 23-36

Menerault Ph. (1993), « Les effets territoriaux d'un outil de financement des transports publics : le versement-transport », Transports Urbains n78, pp. 21-24.

Menerault Ph. (1995), «Approche géo-institutionnelle des relations entre transports publics urbains et territoires à l'échelle du Nord-Pas-de-Calais », Hommes et Terres du Nord, 1995-3, pp. 161-166.

Menerault Ph., Stransky V. (2002), « Représentations graphiques : où est passée l'intermodalité ? Une approche nationale, internationale et locale des plans de réseaux de transports collectifs en milieu urbain », $49 \mathrm{p}$.

Menerault Ph. (2005), "Réforme territoriale et dynamique de l'intercommunalité dans les transports collectifs urbains : une approche diachronique ", in Gallez C., Menerault P. (dir), Recomposition intercommunale et enjeux des transports publics en milieu urbain, Rapport PREDIT, convention ADEME-INRETS n ${ }^{\circ}$ C03-13, pp. 15-23.

Nonn H. (2000), «Déclinaisons sur la recomposition des territoires dans le cadre français », Revue de géographie de l'Est, Tome XL n, pp. 175-181.

Offner J.M. (2002), "Les transports urbains: entre secteurs, réseaux et territoires ", in Annuaire 2002 des collectivités locales, Grale, éditions du CNRS, pp. 169-184.

Offner J.-M. (2003), «L'élaboration des Plans de Déplacements Urbains de la loi sur l'air de 1996 », Rapport de recherche du Latts, ENPC.

Offner J.M. (2006), Les plans de déplacements urbains, PREDIT, coll. Le point sur, Ed. La documentation française, $96 \mathrm{p}$.

Ollivier-Trigalo M. (dir.) (2007), «Six régions à l'épreuve des politiques de transport: décentralisation, régionalisation ferroviaire et différenciation territoriale », Rapport INRETS $\mathrm{N}^{\circ} 273,233 \mathrm{p}$.

Pinson G. (2002), « Political government and governance : strategic planning and the reshaping of political capacity in Turin », International Journal of Urban and Regional Research, vol. 26, $n^{\circ} 3$.

Pinson G. (2006), «Projets de ville et gouvernance urbaine. Pluralisation des espaces politiques et recomposition d'une capacité d'action collective dans les villes européennes », Revue française de science politique, vol.56, $\mathrm{n}^{\circ} 4$.

Pinson G. (2010), "La gouvernance des villes françaises. Du schéma centre-périphérie au régimes urbains $"$, Métropoles, $n^{\circ} 7-2010,17 \mathrm{p}$.

Richer C. (2005), « Les transformations récentes de l'intercommunalité en matière de transport ", in Gallez C., Menerault Ph. (Dir.), Recomposition intercommunale et enjeux des transports publics en milieu urbain, Rapport PREDIT, convention ADEME-INRETS nC03-13, pp. 25-49.

Richer C., Menerault Ph. et al. (2007), Les intercommunalités et les départements face aux enjeux des transports publics, Coll. Dossier n¹88, Ed. CERTU, 88 p.

Richer C. (2009), «Transports publics et recompositions territoriales dans le Nord-Pas-deCalais », Mappemonde, $\mathrm{n}^{\circ}$ 95, 3-2009.

Roseau N. (2010), "Mobile en métropole : une condition durable ? », Urbanisme, HS n³6, pp.59-61

Stone C. (1989), Regime politics : gouverning Atlanta, 1946-1988, University Press of Kansas, Lawrence.

Subra Ph. (2007), Géopolitique de l'aménagement du territoire, collection Perspectives Géopolitiques, Ed. Armand Colin, 327 p.

Vanier M. (2000), "La recomposition territoriale, un grand débat idéal », Espace et Société, $\mathrm{n}^{\circ} 96$, pp. 125-143.

Vanier M. (2008), Le pouvoir des territoires. Essai sur l'interterritorialité, Economica, Anthropos, $160 \mathrm{p}$. 
Wiel M. (1994), «Comment articuler la planification des déplacements et les stratégies urbaines ? », Recherche Transports et Sécurité n44, 1994, pp. 59-70

Wiel M. (1999), La transition urbaine ou le passage de la ville pédestre à la ville motorisée, Edition P.Mardaga, 149 p.

Wiel M. (2010), « Métropole et mobilité : les contradictions métropolitaines », Communication aux $15^{\text {ème }}$ Université d'Eté du Conseil Français des Urbanistes, Strasbourg, 25-26-27 août 2010.

${ }^{1}$ Loi n ${ }^{\circ} 2000-1208$ du 13 décembre 2000 relative à la solidarité et au renouvellement urbains, dite "loi SRU ».
Marc Wiel (2010) prend l'exemple de la ville transfrontalière pour illustrer la nécessaire coopération des
politiques publiques : dans ces villes, il n'y a pas d'unité de pouvoir donc pas de capacité d'arbitrer. Ces
métropoles transfrontalières telles Genève ou Strasbourg ont l'obligation de négocier. Par extension, toutes les
métropoles sont quelque part transfrontalières donc soumis aux mêmes enjeux de gouvernance, que ce soit entre les intercommunalités centrales et périurbaines (Estèbe, 2008) ou avec d'autres échelons d'administration.

${ }^{3}$ Expression de Borraz, John, 2004

${ }^{4}$ Comité pour la réforme des collectivités locales (2009), Il est temps de décider, Rapport au président de la République, $174 \mathrm{p}$.

${ }^{5}$ Le premier article du décret-loi du 30 octobre 1935 précise que « les départements, communes, chambres de commerce et établissements publics peuvent se regrouper sous forme de syndicats pour l'exploitation, par voie de concession, de services publics représentant un intérêt pour chacune des personnes morales en cause ».

${ }^{6}$ A titre d'exemple, à Dunkerque, le Syndicat Mixte des Transports Publics est dissout suite au retrait du conseil général du Nord en 1998. La Communauté Urbaine gère dorénavant seule les transports publics urbains Dunkerquois. A Lyon et à Lille, le retrait progressif des conseils généraux des syndicats mixtes de transport urbain est aussi d'actualité.

7 La nouvelle loi de réforme des collectivités territoriales prévoit la disparition de la clause de compétence générale pour les Départements à compter du 1er janvier 2015: ceux-ci ne pourront plus participer à l'organisation d'un réseau de TCU lorsqu'il y a un PTU.

${ }^{8}$ Les syndicats mixtes SRU créés depuis 2008 et non intégrés à cette étude, sont: le Syndicat Mixte des Transports «Le Fil Vert»; le Syndicat Mixte des Transports Suburbains de Reims; le Syndicat Mixte des Transports des Bouches du Rhône; et enfin, le Syndicat Mixte Intermodal Régional des Transports (SMIRT associant la Région Nord-Pas-de-Calais, les 2 conseils généraux et 12 AOTU).

${ }^{9}$ Le Syndicat Mixte des Transports en Commun Suburbains dans la région de Nancy (SMTCSN), substitué au département de Meurthe-et-Moselle s'agissant de la partie suburbaine de certaines lignes, existe depuis 1981.

${ }^{10}$ Cette remarque est aussi vrai en dehors du domaine des transports publics, puisque le nombre de syndicats mixtes (de diverses objets) a plus que doublé entre 1999 et 2005 (de 1454 à 3029).

${ }^{11}$ A titre d'exemple, le conseil général du Nord a dû céder la place qu'il partageait avec Lille Métropole Communauté Urbaine (LMCU) pour l'organisation des transports urbains afin de pouvoir adhérer au syndicat mixte SRU régional en création à un seul titre d'AOT des TCNU et non au double titre d'AOT des TCU de Lille et des TCNU du Nord.

${ }^{12}$ Autant imaginer une généralisation du VT pour valider l'abolition des limites urbain/non-urbain à une échelle donnée (du syndicat mixte SRU, du département ou de la région) comme le suggère Jean-Paul Bachy : lors de son intervention lors du colloque GART le 9 novembre 2010, le Président du Conseil Régional de ChampagneArdenne, assure qu'il faudrait « généraliser le versement-transport » à l'ensemble de la Région.

${ }^{13} \mathrm{Au}$ même titre que les questions posées par Philippe Menerault et Vaclav Stransky à propos des représentations graphiques des plans de réseaux de transport urbain (Menerault, Stransky, 2002). 